\title{
Compositional Genome Contexts Affect Gene Expression Control in Sea Urchin Embryo
}

\author{
Abdullah Al Mahmud, Gabriele Amore*, Giorgio Bernardi
}

Stazione Zoologica "Anton Dohrn" Napoli, Villa Comunale, Napoli, Italy

\begin{abstract}
Gene expression is widely perceived as exclusively controlled by the information contained in cis-regulatory regions. These are built in a modular way, each module being a cluster of binding sites for the transcription factors that control the level, the location and the time at which gene transcription takes place. On the other hand, results from our laboratory have shown that gene expression is affected by the compositional properties (GC levels) of the isochores in which genes are embedded, i.e. the genome context. To clarify how compositional genomic properties affect the way cis-regulatory information is utilized, we have changed the genome context of a GFP-reporter gene containing the complete cisregulatory region of the gene spdeadringer (spdri), expressed during sea urchin embryogenesis. We have observed that GC levels higher or lower than those found in the natural genome context can alter the reporter expression pattern. We explain this as the result of an interference with the functionality of specific modules in the gene's cis-regulatory region. From these observations we derive the notion that the compositional properties of the genome context can affect cis-regulatory control of gene expression. Therefore although the way a gene works depends on the information contained in its cis-regulatory region, availability of such information depends on the compositional properties of the genomic context.
\end{abstract}

Citation: Mahmud AA, Amore G, Bernardi G (2008) Compositional Genome Contexts Affect Gene Expression Control in Sea Urchin Embryo. PLoS ONE 3(12): e4025. doi:10.1371/journal.pone.0004025

Editor: Jean-Nicolas Volff, Ecole Normale Supérieure de Lyon, France

Received August 14, 2008; Accepted November 21, 2008; Published December 29, 2008

Copyright: (C) 2008 Mahmud et al. This is an open-access article distributed under the terms of the Creative Commons Attribution License, which permits unrestricted use, distribution, and reproduction in any medium, provided the original author and source are credited.

Funding: This work was funded in part with a MIRG 036543 Grant, FP6th. The funders had no role in the design and conduct of the study, in the collection, analysis, and interpretation of the data, and in the preparation, review, or approval of the manuscript.

Competing Interests: The authors have declared that no competing interests exist.

*E-mail: gabriele.amore@szn.it

\section{Introduction}

Investigations carried out in our laboratory many years ago established that genomes of vertebrates (and other eukaryotes) are mosaics of isochores, megabase-sized DNA regions, that are compositionally fairly homogeneous and belong in a small number of families covering a broad GC (the molar ratio of guanine and cytosine) range $[1,2]$. This compositional compartmentalization is correlated with a number of both structural and functional properties (chromatin structure, genes and repeats distribution, introns and UTRs size; gene expression levels, replication timing, recombination). The compositional correlation between coding and flanking sequences established the concept of genome as an integrated ensemble and rejected the widely accepted view of genes being distributed at random in non-coding "junk" DNA [3]. The functional relevance of these observations was demonstrated by the fact that stable integration and appropriate expression of mammalian retroviruses was possible only in host genomic contexts of similar composition (isopycnic localization; [4,5,6,7,8]) namely when the compositional correlations that host genes have with their genome context is fulfilled [1]. Regional genomic properties are therefore relevant to the functionality of genes.

On the other hand, single gene-level studies have shown how gene expression is controlled through the utilization of the information contained inside cis-regulatory regions. These are composed of modules, clusters of binding sites for the factors that regulate transcription. Transcriptional control relies on the conditional activation of factors so to respond to the need of activating certain genes at specific times and in certain cells and to transcribe them at appropriate levels [9].

In the present study, we investigated if and how the compositional properties of the DNA surrounding the cis-regulatory region of a gene (the genome context) can affect its mode of work. We have utilized the Strongylocentrotus purpuratus sea urchin embryo, where we have monitored the effects of altering the compositional properties of the genome context on the expression pattern of a green fluorescent protein (GFP) reporter containing the complete cis-regulatory region of the spdri gene [10]. Activation of spdri happens first (from 13 to $24 \mathrm{~h}$ of development) in the primary mesenchyme cells (PMCs, the cells that build the embryonic skeleton). Afterward (from the onset of gastrulation) expression of the gene switches to the oral ectoderm $(\mathrm{OE})$ where it is maintained throughout development. We have recently isolated a genomic DNA fragment (“4.7IL": -3456/+389) that can faithfully replicate the gene's expression pattern. This has been verified in experiments in which this DNA fragment is fused together with a GFP coding sequence, the resulting construct (4.7ILGFP) is injected in sea urchin zygotes and the expression pattern is observed in the developing embryos. In these experiments, 4.7ILGFP is expressed in PMCs from 13 to $24 \mathrm{~h}$ and in the oral ectoderm afterward, with essentially no ectopic expression.

While a detailed description of the structure and mode of work of this cis-regulatory region can be found elsewhere [7], a summary of it is given in Fig. 1, where a dissection of 4.7IL is provided with a description of the function of the regulatory modules identified in it. Expression in the PMCs is obtained through the activity of three modules. A proximal module (Ubiq+; 1.8; fig. 1A) that responds to 


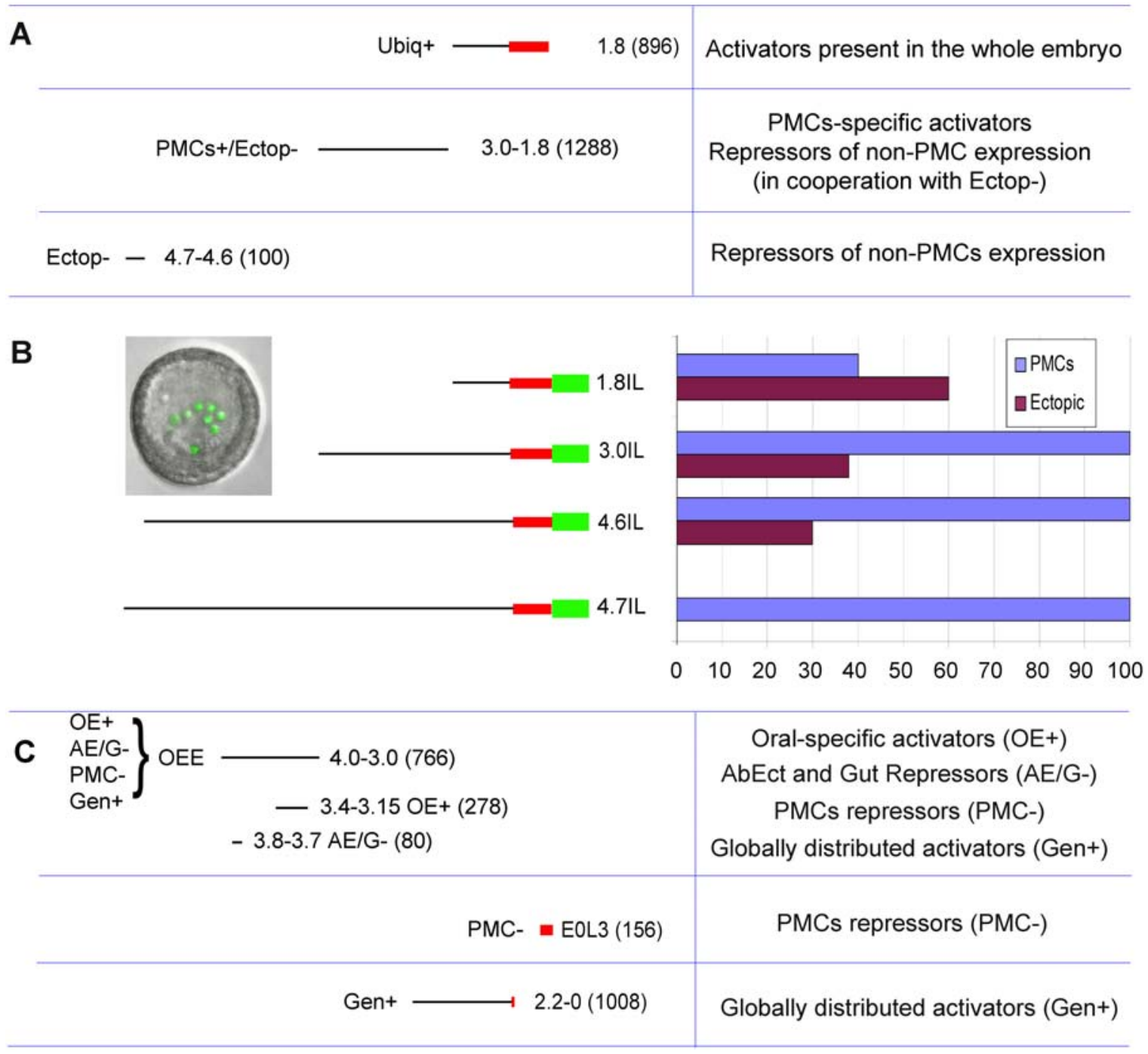

D

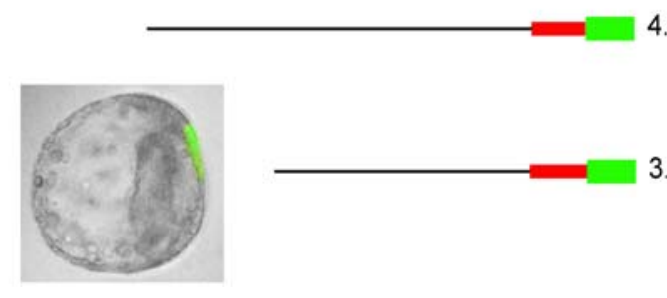

4.0IL

3.0IL

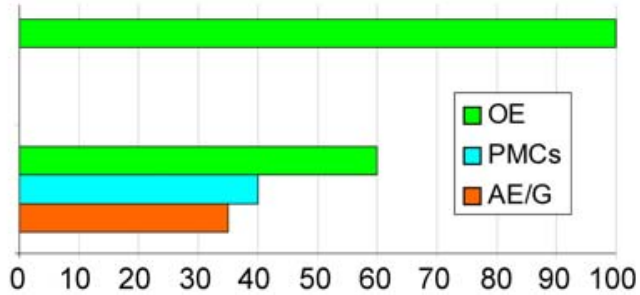

Figure 1. Summary of spdri's cis-regulatory region [7]. All constructs are depicted with a black horizontal line, which represents genomic DNA, and a red box, which is spdri's first exon. Constructs 4.7IL-GFP, 4.6IL-GFP, 4.0IL-GFP, 3.0IL-GFP and 1.8IL-GFP were obtained by fusing a GFP coding cassette downstream of the first exon of spdri (GFP cassette is indicated with a green box in the diagrams); the function of the OEE module was studied by cloning it directly into the EpGFP vector. To test the function of the EOL3L fragment, a version of 4.7IL-GFP where this element was deleted was produced [10]. Abbreviations are as follows. OE: oral ectoderm; AE: aboral ectoderm; G: gut. (A) A table showing (on the left) the PMCs modules. Here, the name of each module is given according to the function assigned (on the left of each module). Numbers on the right of each module are used to indicate the position of the extremities of the module with respect to the transcriptional start site. The size in nucleotides of each module is given in parenthesis. On the right part of the table, a brief description of the regulators operating on each module is provided. (B) A picture of a live $24 \mathrm{~h}$ embryo with GFP fluorescent PMCs (green cells) is shown on the left. The vegetal pole is at the bottom. The expression pattern of the indicated constructs is reported as observed in $24 \mathrm{~h}$ live embryos. At this stage, GFP is seen in PMCs (blue bars), at ectopic locations (purple bars), or both. (C) Regulatory modules responsible for oral ectoderm expression are shown similarly to $(A)$. The four functions assigned to the OEE are indicated. OE+: oral specific activation; $\mathrm{AE} / \mathrm{G}-$ : repression of expression in aboral ectoderm and gut; PMC -: repression of expression in PMCs; Gen+: activation in non oral territories. The position of $\mathrm{AE} / \mathrm{G}-$ and $\mathrm{OE}+$ is explicitly indicated. (D) A picture of a live $48 \mathrm{~h} \mathrm{embryo}$ with GFP fluorescence in the oral ectoderm is show on the left. Oral ectoderm is on the right and the embryo is shown from the side. Invaginated gut is visible in its length. The vegetal pole is at the bottom. The expression pattern of the indicated constructs is reported as observed in $48 \mathrm{~h}$ live embryos; the consequence of removing the OEE module from 4.0IL construct is illustrated. Expression in the different territories is indicated according to the legend provided.

doi:10.1371/journal.pone.0004025.g001 
factors present in all the cells of the embryo; when this module is tested alone (construct 1.8IL-GFP; fig. 1B), GFP expression is observed in PMCs as well as in all other embryonic territories, with no bias toward a specific one. A second module (PMC+/ Ectop-; 3.0-1.8; Fig. 1A) is controlled by PMCs-specific activators and by non-PMCs repressors (used to prevent ectopic expression). Addition of this module to 1.8IL results in construct 3.0IL (Fig. 1B), which drives GFP expression in PMCs of about $100 \%$ of the embryos, but maintains some ectopic expression $(30 \%$ to $40 \%$ ). Finally a distal module (Ectop-; 4.7-4.6; fig 1.A), is needed to completely eliminate ectopic expression, through the binding of transcriptional repressors present in cells other than PMCs. When this module is removed from 4.7IL (and construct 4.6IL is obtained; fig. 1B) the percentage of embryos expressing in the PMCs is maintained to about $100 \%$, but the number of embryos expressing at ectopic locations remains significant (about $30 \%)$. It is likely that this module and the $3.0-1.8$ module interact to ensure complete elimination of ectopic expression.

At $48 \mathrm{~h}$, the $4.0 \mathrm{IL}$ construct $\left(833 \mathrm{nt}\right.$ shorter at its $5^{\prime}$-terminus than 4.7IL; fig. 1C) ensures correct expression in the oral ectoderm (Fig. 1D). This is obtained through the "OE+" activity of the Oral Ectoderm Enhancer (OEE; fig 1C), which responds to oral ectoderm-specific activators. This module can promote transcription in the oral ectoderm after $24 \mathrm{~h}$, if cloned alone into a Ep-GFP vector, which carries a sea urchin basal promoter [11]. When the OEE is removed from 4.0IL (and construct 3.0IL is obtained; fig. 1C), oral expression drops from $100 \%$ to $60 \%$. The OEE also binds repressors that are required to terminate expression in PMCs after $24 \mathrm{~h}$ ("PMC-" activity). This repression is obtained through a cooperation with the activity localized at the $3^{\prime}$ portion of spdri's first exon ("E0L3"; fig. 1C). The OEE is also needed to prevent ectopic expression in embryonic aboral ectoderm and gut ("AE/G-" activity), as deletion of it increases expression in these territories conspicuously. Finally the OEE possess a general enhancer activity $(\mathrm{Gen}+)$; this is responsible for the expression of the reporter gene in non-oral ectoderm territories and becomes evident when the repressor portions of the module are removed. A similar "Gen+" activity is shown by the 3.0IL construct at this time; this activity is localized in the most proximal part of this genomic DNA fragment (the 2.0-0 fragment).

In the present work we have utilized this information to interpret the results of experiments in which we changed the composition of the genome context of construct 4.7IL-GFP, so that its GC level was different than that of the endogenous spdri gene's genomic context. As this kind of manipulation resulted in striking alteration of the expression pattern of our construct, we derived an indication that the compositional properties of the genome context can affect the way the information contained inside a cis-regulatory region is utilized.

\section{Results}

\section{Construct molecules intersperse with carrier during concatemer formation}

The spdri's cis-regulation model presented in the previous section is based on the result of transgenesis experiments performed by microinjection. In a typical experiment, constructs are introduced in sea urchin zygotes together with restriction enzyme-digested genomic DNA, which is used as carrier (in the following referred as whole genomic DNA, WGD). It is well documented that in these conditions a construct-carrier concatemer is produced by the ligases present in the egg, which integrates in the genome after a few cell divisions and contains several hundred molecules of construct. It is assumed that in the conditions used in a standard transgenesis experiment, construct molecules are interspersed with the carrier fragments in the concatemer and that the spacing between each construct molecules depends on the molar ratio carrier: construct [12].

Because it was critical for the correct interpretation of the data presented in this report, we sought to verify that incorporation in fact happened so that our construct molecules (4.7Il-GFP) would be evenly interspersed, within the concatemer with no significant occurrence of construct "concatenation" (i.e. the formation construct-construct tandems). To this aim we analysed the genomic DNA of transgenic embryos and compared the amplification signal obtained by using a couple of primers specific for the GFP coding sequence with that of a couple of primers amplifying the "junction" that would form between two concatenated construct molecules, by way of real-time quantitative PGR (qPGR). This comparison allowed us to determine how many concatenation events would occur per incorporated construct molecule. The results we obtained are shown in Fig. 2.

When injected together with whole genomic DNA (4.7IL+WGD in Figure 2B), the construct-construct concatenation amplification signal amounted to $4-5 \%$ of the number of integrated construct molecules. Because each construct molecule can concatenate in two different orientations with respect to the next one, this means that in a standard transgenesis experiment $10 \%$ of construct molecule concatenate at the most. On the other hand when no carrier was used (the amount of construct molecules was in this case adjusted to reach the mass of DNA necessary to achieve incorporation), the occurrence of concatenates would increase about ten times (40-45\%). Therefore in these conditions almost all construct molecules (up to $90 \%$ ) concatenated. When a specific sequence (HGC4 in Figure 2B, or others not shown here) would be used as carrier, construct concatenation level would be the same as that of 4.7IL+WGD. In this case we could also measure construct-carrier concatenation and observe that it was at such a level that almost all construct molecules would be flanked by carrier molecules (again about 90\%, considering two possible orientations for the carrier molecules). Therefore when carrier is utilized, this allows construct molecules interspersion within the concatemer and very low occurrence of construct concatenation is observed. More importantly, the GC level of the genome context of our construct molecules is necessarily that of the DNA carrier utilized.

Compared to other genomes such as those of vertebrates, the sea urchin genome presents a low level of heterogeneity. This has been already reported in previous investigations [1] and it has been confirmed by the analysis of the sequence data from the Sea Urchin Genome Consortium [13]. The spread of the compositional distribution, as calculated on the basis of the sequence information, is between $34 \%$ and $40 \%$ GG if scaffolds equal to or larger than $25 \mathrm{~kb}$ are put in $0.5 \%$ GC bins. These scaffolds show a 1.8-fold increase in gene density with increasing GC-levels (manuscript in preparation). Most of the genomic DNA has a GC level comprised between 36 and 39\% GC and the average value of the genome is $37.9 \%$. This means that in a standard microinjection procedure, the compositional genome context of most of the construct molecules will be included in this range.

As the goal of our research was to investigate the effects of changing the GC level of the genomic context of our GFP constructs, we reasoned that we could obtain this by changing the GC level of our carrier DNA. Therefore we utilized two different DNA fractions from $\mathrm{CsCl}$ shallow gradient centrifugation of sea urchin sperm DNA, as carrier. These were recovered from the GC-poorer and the GC-richer portions of the gradient and their GC level was lower and higher than average GC level of sea 

GFP primers
Construct-construct primers
construct-carrier (HGC4) primers

C

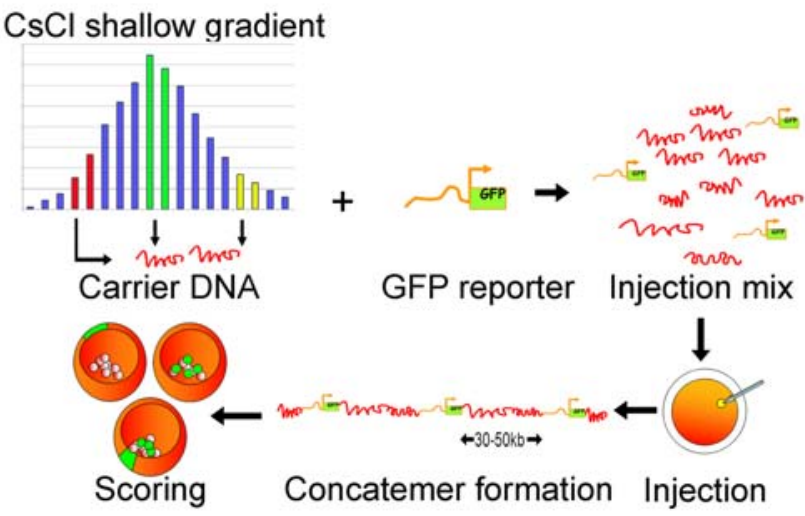

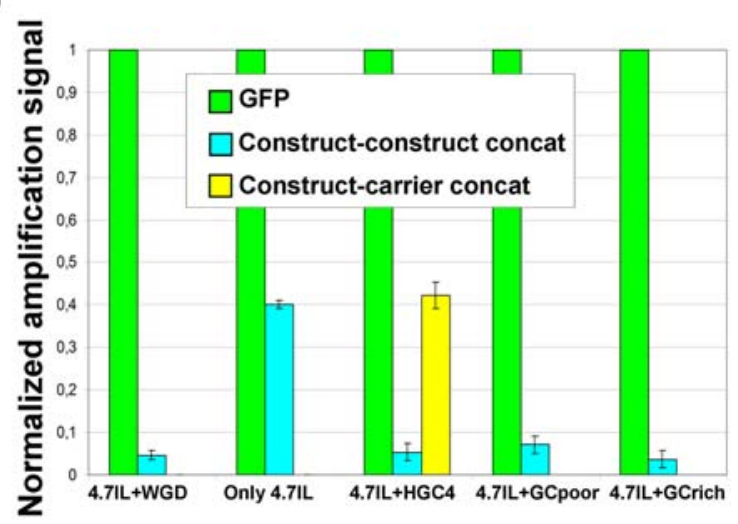

D

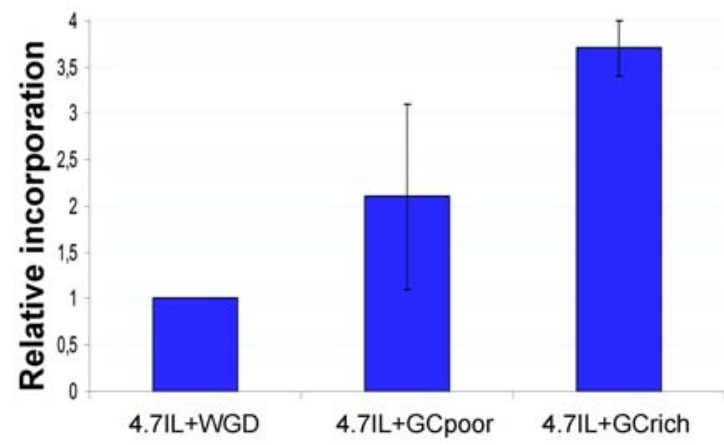

Figure 2. Integrated construct molecules intersperse within the concatemer. (A) A schematic view of construct 4.7IL-GFP showing the upstream portion, the first exon (red) and the GFP coding sequence (green). The portion between the upstream regulatory modules and the first exon is shown with a dashed line (not in scale). Primer couples used for quantitative PCR are shown below: in green primers used to amplify the GFP coding sequence: these two primers will only give an amplification signal if a construct-construct concatenate forms; in light blue those utilized to amplify at the junction between two adjacent construct molecules; in pale yellow those utilized to amplify at the junction between construct and carrier (HGC4 in the figure) molecules. The distance between primers is maintained around $120 \mathrm{nt}$. Carrier molecule is not shown. (B) Constructconstruct (or construct-carrier) concatenation is measured Vs construct incorporation. The amount of construct molecules is measured by the level of the amplification of GFP. Construct incorporation level is equated to 1 in each experiment and the level of concatenation is measured by comparing the $\mathrm{Ct}$ value obtained for the amplification of construct-construct (or construct-carrier) amplification to that of construct incorporation. This allows to asses how many times concatenation occurs per each construct molecule incorporated in the genome. About $10 \%$ of the incorporated construct molecules concatenate when the WGD, a specific sequence (HGC4) or the DNA from shallow gradient fractions is used as carrier. In each experiment 100-150 embryos are utilized. Experiments were repeated using at least two different batches of embryos. (C) In order to prepare carrier DNA of chosen GC level, S. purpuratus genomic DNA was extracted and fractionated by $\mathrm{CsCl}$ shallow gradient ultracentrifugation. DNA from fractions at average GC (37.9\%; green bars), GC-poor (34-35\%; red bars) or GC-rich (about 40\%; yellow bars) portions was utilized as carrier, in experiments where the 4.7IL-GFP construct was injected in zygotes. Upon concatemer formation, incorporation of the construct in the genomic DNA is obtained in a defined compositional context. (D) Incorporation of 4.7IL construct molecules is equal or higher when "GC-poor" or "GC-rich" carrier DNA is used compared to WGD. All the results presented here were verified in at least three separate experiments where independent batches of embryos were utilized.

doi:10.1371/journal.pone.0004025.g002

urchin DNA (about 34-35\% GC for "GC-poor" and about 40\% GC for "GC-rich"; Fig. 2C). Here we show that when DNA from these fractions is used as carrier, construct-construct concatenation occurs at levels which are similar to those observed in a standard injection (Fig. 2B). Therefore construct molecules intersperse within the concatemer in these conditions. Furthermore we show that incorporation of construct molecules is not hampered by the use of such carrier. In comparison with standard injection procedure, the level of incorporation of construct molecules is similar or even higher when the "GC-poor" and "GC-rich" carrier are utilized (Fig. 2D).

Changes in the compositional genome context affect cisregulatory control of gene expression

As mentioned above, by using fractions of genomic DNA separated by $\mathrm{CsCl}$ shallow-gradient ultracentrifugation as a source for our carrier DNA we could embed our construct molecules in a genome context whose GC level could be manipulated. This allowed us to assess if the GC level of the genome context could affect the mode of work of our cis-regulatory region. In the following we present and discuss the results obtained when injecting 4.7ILGFP in $S$. purpuratus zygotes (Fig. $3 \mathrm{~A}-\mathrm{I}$ ). We have also performed experiments in which we have utilized another version of the construct, D-GFP, which extends $4.7 \mathrm{IL}$ by $1 \mathrm{~kb}$ at its $5^{\prime}$ terminus and $897 \mathrm{bp}$ at its $3^{\prime}$ terminus and includes the first intron and the second exon. The observations we made are consistent with those performed when injecting 4.7IL-GFP (not shown).

When injections were performed using as carrier the DNA recovered from the main fraction of the density gradient (GC level around $37.9 \%$ ), the expression pattern of the injected construct was identical to that observed in a typical injection experiment (and to that of the endogenous spdri gene). However when fractions at the 
A

4.7IL Control (24h)

$\begin{array}{lllllllllll}0 & 10 & 20 & 30 & 40 & 50 & 60 & 70 & 80 & 90 & 100\end{array}$

$4.7 \mathrm{LL}+\mathrm{GC}-$ poor $(24 \mathrm{~h})$
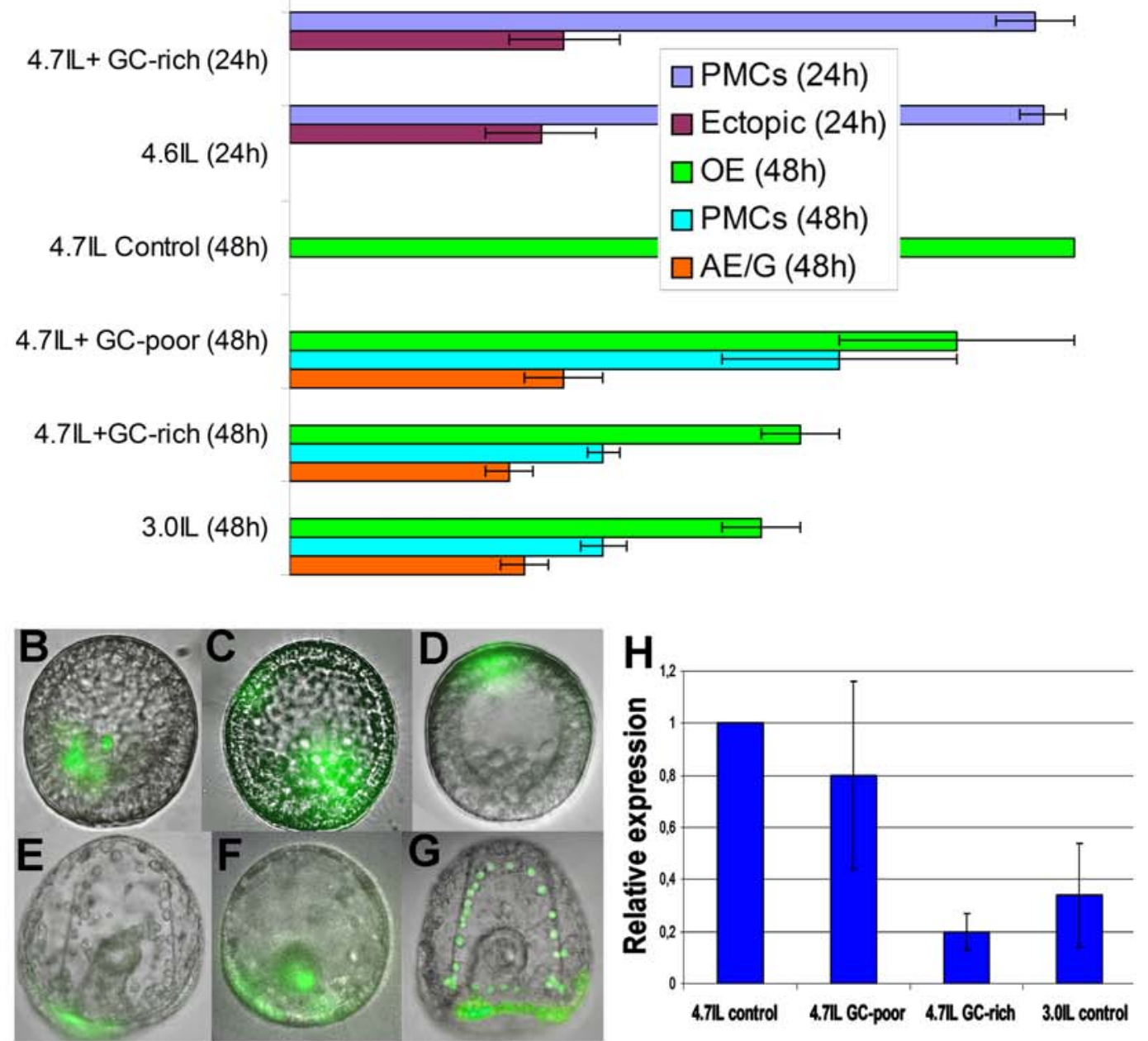

I
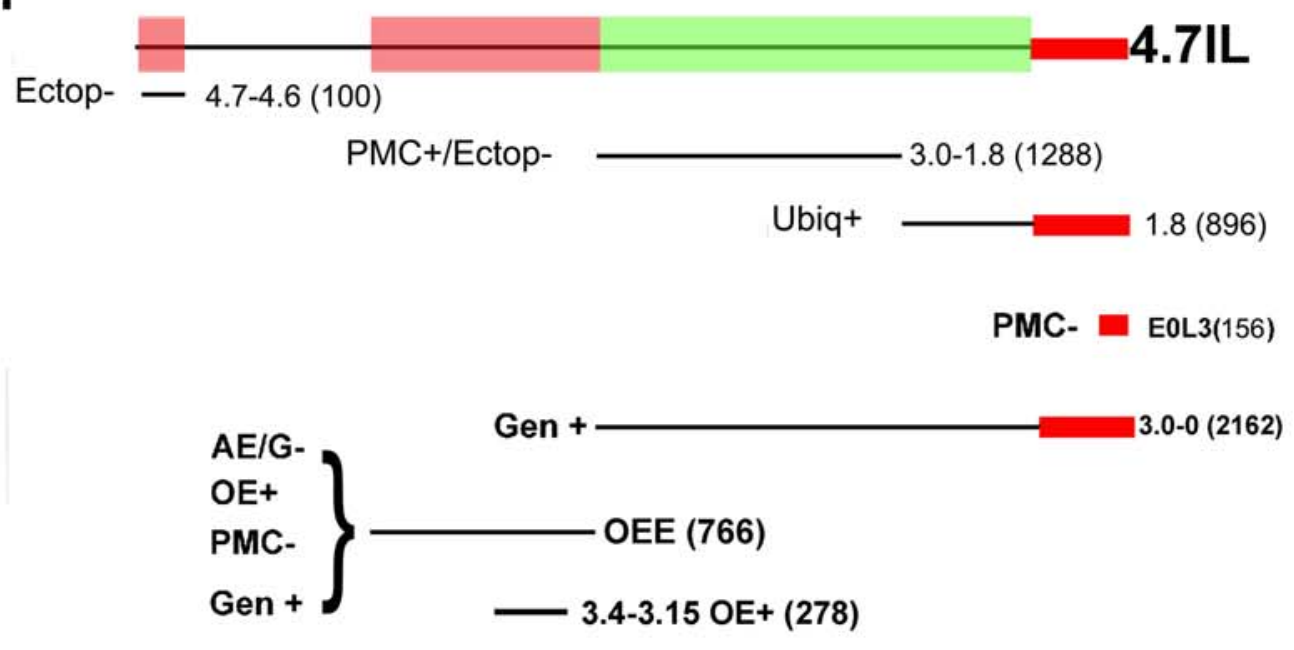

- 3.8-3.7 AE/G- (80) 
Figure 3. Changing the genomic context's GC level interferes with control of gene expression. (A) After injection, embryos were scored for GFP fluorescence at the times indicated in parentheses. Scoring results are given in a histogram form. Percentages of expression in the different territories are indicated according to the legend. Note that in the same embryos expression can happen in more than one territory. "Control" is used to indicate results from embryos injected with non-fractionated carrier DNA, or with carrier DNA from the central fraction of the gradient; in both cases the same result was obtained and the construct expressed appropriately. "GC-poor" and "GC-rich" indicate embryos injected with GC-poor, or GC-rich carrier DNA respectively. Abbreviations are as in fig. 1. (B-G) Representative pictures of injected embryos at $24 \mathrm{~h}$ (B-D) or $48 \mathrm{~h}$ (E-G; embryos are shown from the vegetal pole; in this view the gut appears in cross section and the PMCs form a chain around it). GFP fluorescence is present only in PMCs or oral ectoderm (B and E; control embryos), PMCs and ectopic locations (C) or in just at ectopic location (D); oral ectoderm and ectopic locations (gut in F and PMCs in G). (H) 4.7IL-GFP transcriptional output is measured at $48 \mathrm{~h}$ in the different injection conditions indicated. Transcriptional output measured upon injection with WGD is taken as reference and equated to 1. (I) 4.7IL-GFP is represented and aligned with its cisregulatory modules. Red and green boxes over the length of 4.7IL-GFP indicate regions of the cis-regulatory DNA whose function is interfered with or left unaffected, upon alteration of the genome context. Abbreviations are as in Fig. 1. Note that upon injection with "GC-poor" carrier DNA the interference on the OEE function is mostly limited to its $5^{\prime}$ portion, where the "AE/G -" element is located.

doi:10.1371/journal.pone.0004025.g003

lowest and highest extremes of the compositional distribution were used, striking alterations of the expression pattern were observed. These were monitored and scored as percentages of embryos expressing GFP in the different territories at two different times: $24 \mathrm{~h}$ (when spdri is normally expressed in PMCs) and $48 \mathrm{~h}$ (when spdri is normally expressed in the oral ectoderm). The results obtained are reported in Fig. 3A. In each experiment about 100 embryos were scored and three or more different batches of embryos were used in independent experiments.

At $24 \mathrm{~h}$ almost all the expressing embryos correctly showed GFP-fluorescence in the PMCs. However, a conspicuous fraction of them displayed GFP-fluorescence also at ectopic locations: in $46 \%$ or in $33 \%$ of the cases (when GC-poor or GC-rich carrier DNA was used respectively) expression was observed in nonPMCs territories with no clear bias toward a specific region of the mesenchyme blastula stage embryo. This expression pattern was highly reminiscent of that observed upon injection of construct 4.6IL-GFP (Fig. 3A), which misses the distal repressor element "Ectopic-". Therefore we interpret the observed result as if the function of this repressor module is abolished when the construct is incorporated in a GG-poor or GC-rich genomic context. As all the embryos express in PMCs, the function of the "PMC+/Ectop-" module has been maintained intact in these conditions (Fig. $3 \mathrm{I}$ ).

At gastrula stage, expression in the oral ectoderm was reduced from $100 \%$ of the controls, to $85 \%$ or $65 \%$ in GC-poor and GCrich respectively. In the case GC-poor fractions were used, we observed a stronger variability in the amount of oral ectoderm expression when comparing different batches. In all the experiments expression in the aboral ectoderm and gut was observed (35\% in low-GC and 28\% in high-GC) as well as permanence of GFP fluorescence in PMCs $70 \%$ or more in low-GC, with high degree of variability and $40 \%$ in high-GC). These expression pattern could be compared with that of construct 3.0IL-GFP (Fig. 3 A), which lacks the entire OEE module. From this comparison it appears that in both situations (GC-poor or GC-rich genomic contexts) the function of the OEE module is interfered with (Fig. $3 \mathrm{I}$ ). When high-GG carrier DNA is utilized the function of the entire OEE module is abolished in a very consistent way. However when low-GC carrier DNA is utilized the extent of such interference is variable and depending on the batch the function of the OEE can be either completely or partially lost.

It is possible that the observed effects would be due to the presence of enhancers fortuitously "trapped" in the carrier DNA. Such occurrence is to be excluded based on the results of the scoring performed at $48 \mathrm{~h}$. In fact if extra enhancers would be present in the carrier, these would add extra domains of expression without affecting expression in the $\mathrm{OE}$, which should be always maintained in $100 \%$ of the expressing embryos. Nonetheless, to make sure that the observed behavior was not due to some non-specific effect of the carrier, or to the presence of extra enhancers, GFP vectors bearing just the basal promoter (EpGFP) were used in parallel control experiments. In this case no or minimal expression of the vector was observed. In another series of control experiments, a reporter containing the minimal enhancer "Y2Y4" from the sea urchin gene spcyclophillin [14] was used. The latter is a 280 bp module that only responds to two positive inputs, provided by the PMCs-specific activators spdri and sp-ets1 [15] and drives expression of its GFP reporter only in these cells. When this reporter was used for control, expression could only be seen in PMCs, independently of the carrier DNA used (not shown).

Finally we measured the transcriptional output of 4.7IL at $48 \mathrm{~h}$ and in the different injection conditions. The results presented in Fig. $3 \mathrm{H}$, clearly show that transcription was never significantly higher than that observed upon standard injection conditions. In particular the transcriptional output of 4.7IL in GG-rich contexts was similar to that of the 3.0IL construct (injected with WGD for control). Therefore we could conclude that in GC-rich context a complete interference with the OEE module was obtained. On the other hand, when the low-GC carrier DNA was used, the transcriptional output showed a greater variability (although never significantly higher than that observed with WGD). We interpret this result as if in this case the interference with the functionality of the OEE is mostly limited to its $\mathrm{AE} / \mathrm{G}$ - portion (which lies at the $5^{\prime}$ of the OEE) and affects only marginally the functionality of the $\mathrm{OE}+$ element inside the OEE module. In any case these results show that no extra enhancer was trapped in the carrier utilized.

Based on these results we conclude that the occurrence of ectopic expression, observed in the experiments with 4.7IL, was due to an interference with the functionality of specific regulatory modules inside spdri's cis-regulatory region, not to the operation of extra enhancers present in the carrier DNA. Finally, all the effects described were not observed when the GsCl DNA fractions closer to the central part of the gradient were used. Therefore the effect observed is dependent on the GG level of the carrier DNA used.

\section{Discussion}

The results described above give us the opportunity to sketch a picture of the relationship between cis-regulatory control and GC level of the genomic context. In the experiments described here, expression of our trans-gene conforms to the information contained inside its cis-regulatory region. However when the compositional genome context of our construct is altered, only part of this information is available and the observed alterations in the expression pattern are a direct consequence of this. Here, we have reported that a complete interference (at $24 \mathrm{~h}$ ) with the functionality of a distal repressor module (4.7-4.6) is obtained both when GCpoor as well as GC-rich carrier DNA is used. The results obtained at $48 \mathrm{~h}$ show that this interference extends to the more proximal module OEE. This module seems to be completely (in GG-rich) or partially affected (in GC-poor) in the altered genomic contexts. The 
observed interference spreads from the most distal portions of the cis-regulatory region toward more proximal ones, affecting at least $1 \mathrm{~kb}$ of DNA (that is the distance between 4.7 and the middle of the OEE) i.e. is distance dependent. Therefore we conclude that (at least in the case described here) although the way a gene works is dependent on the information contained inside its cis-regulatory region, the availability of such information depends on the properties of the gene's genomic context.

Gene transcription is determined by the particular combination of transcription factors that are present at any given time and place in a cell nucleus. Upon interaction with their cognate binding sites, transcription factors mediate DNA looping so that even distant modules can be brought to interact with the basal transcriptional apparatus [9]. We hypothesize that GC level can affect the properties of genomic contexts' chromatin (condensation, methylation, nucleosome positioning), which in turn might affect the functionality of adjacent cis-regulatory regions. The mechanisms by which this is obtained remain to be elucidated.

In our study we have related precise alterations in the way cisregulatory information is accessed to the GC level of the genome context. From our results we can derive a definition of a "proper" genome context as the DNA with compositional properties that are appropriate to allow the functionality of a gene's cis-regulatory region. Given the direct, measurable effect of genome context's GC level on the way cis-regulatory information is utilized we can imagine that changes in the genomic context's GC level can have important consequences for the functionality of genes. Such changes might result in "genomic diseases" [1,2] where the functionality of genes might be affected even though no specific mutation in the coding or in the regulatory sequence of a gene might be identified.

\section{Materials and Methods}

\section{Embryo culture, microinjection and scoring}

Fertilized eggs were injected with $1-2 \mathrm{pl}$ of a solution containing 250 molecules of reporter construct/pl, following described microinjection and embryo culture procedures [12]. Live embryos were observed under UV light on a Zeiss Axioscope 40; GFP expression in the different cells was assessed and embryos were scored and photographed using a Canon Powershot 6 camera.

\section{$\mathrm{CsCl}$ ultracentrifugation and carrier DNA preparation}

Analytical and preparative ultracentrifugation of sea urchin genomic DNA was performed as described [16]. For preparative purposes 50 to $100 \mu \mathrm{g}$ of genomic DNA were utilized. Fractions were eluted, UV quantified and salts were removed by dropdialysis. To assess the GG content of individual fractions, analytical ultracentrifugation was performed with $1 \mu \mathrm{g}$ of DNA from the fraction (when possible) or from several fractions with a higher DNA content to derive a standard curve; alternatively the GC level was derived from the refraction index of the fraction as in [2]. DNA from fractions to be used as carrier, was digested with Hind III restriction enzyme (this ensured the highest rate of incorporation). Given the carrier to construct ratio utilized in microinjection experiments, a spacing of $30-50 \mathrm{~Kb}$ is expected to exist between each construct molecules, upon concatemer formation and incorporation into the genomic DNA.

\section{References}

1. Bernardi G (2004) Structural and Evolutionary Genomics. Natural Selection in Genome Evolution. The Netherland: Elsevier.

2. Bernardi G (2007) The neoselectionist theory of genome evolution. Proc Natl Acad Sci U S A 104: 8385-8390.

3. Ohno S (1972) So much "junk" DNA in our genome. Brookhaven Symp Biol 23: $366-370$.

\section{Quantitative real-time PCR (qPCR) measurements}

At the appropriate time point 100-150 embryos were collected and processed using the reagents in the Quiagen "All prep DNA/ RNA mini kit" (Qiagen Inc., Valencia, CA). For quantitative PCR, "Fast Start Sybr Green Master" (Roche) was used. Reactions were run on an MJ Research-Biorad Chromo 4 machine, equipped with "Opticon Monitor" analysis software. Each replicate reaction was performed in a total volume of 10 or $15 \mu \mathrm{l}$ using the equivalent amount of 2-3 embryos. For quantitative measurements an arbitrary threshold is set in the linear phase of amplification. The number of cycles necessary to reach the same amplification level (at the threshold) between different samples is converted in the corresponding ratio in the amount of DNA or cDNA. This is given by $1.94^{\Delta \mathrm{Ct}}$ where $\Delta \mathrm{Ct}$ is the difference in the number of cycles needed to reach the threshold between samples. Therefore using transgenic embryos genomic DNA, the level of construct concatenation can be derived by comparing the amplification signal from construct-construct or construct-carrier concatenation with that of GFP coding sequence; incorporation of constructs can be assayed by comparing the GFP signal with that obtained from a single copy gene (SpfoxA). Transcriptional output can be estimated by comparing GFP amplification signal from cDNA between different samples after normalizing for construct incorporation and for the amount of RNA utilized (using the signal from the amplification of $S p z 12$ as described in $[7,14])$. A baseline signal is obtained using DNA from non-injected embryos. Meaningful differences in the level of the amplification signal measured are considered those where $\Delta \mathrm{Ct}=>|1.7|$. because of the chemistry utilized, this correspond to a time-fold change of about 3 or more.

Primers utilized for the experiments described in Fig. 2 were as follows:

Primers for construct-construct concatenation:

pGFPA: 5'-GGGAGGTGTGGGAGGTTTT-3'

q4'7r: 5'-CGTGACTGCTAAGAAAGGATTACG-3'

Primers for construct-HGC4 concatenation:

pGFPA (see above)

H4Jr: 5'-TCTTCGCGTCAACGACTTGT-3'

Primers for GFP, SpfoxA and Spz12 were as in [10,14]

\section{Other procedures}

All other procedures utilized here are standard laboratory protocols. Detailed information on spdri and spcyclophillin cisregulation can be found in $[10,14]$.

\section{Acknowledgments}

We would like to express a special thank to Dr. P. Sordino of SZN, Dr. D. Corona of Dulbecco Telethon Institute Palermo, to Mrs L. Bellavia and Mrs F. Parveen, for discussions and encouragement; to the technical staff of Stazione Zoologica Napoli for help and assistance throughout.

\section{Author Contributions}

Conceived and designed the experiments: GA. Performed the experiments: AAM GA. Analyzed the data: GA. Wrote the paper: GA GB.

4. Zerial M, Salinas J, Filipski J, Bernardi G (1986) Genomic localization of hepatitis B virus in a human hepatoma cell line. Nucleic Acids Res 14: 8373-8386.

5. Rynditch A, Kadi F, Geryk J, Zoubak S, Svoboda J, et al. (1991) The isopycnic, compartmentalized integration of Rous sarcoma virus sequences. Gene 106: 165-172. 
6. Zoubak S, Richardson J, Rynditch A, Höllsberg P, Hafler D, et al. (1994) Regional specificity of HTLV-I proviral integration in the human genome. Gene 143: 155-163.

7. Rynditch A, Zoubak S, Tsyba L, Tryapitsina-Guley N, Bernardi G (1998) The regional integration of retroviral sequences into the mosaic genomes of mammals. Gene 222: 1-16.

8. Arhondakis S, Clay O, Bernardi G (2008) GC level and expression of human coding sequences. Biochem Biophys Res Commun 367: 542-545.

9. Davidson E (2006) The regulatory genome Gene regulatory networks in development and evolution: Elsevier-Academic Press.

10. Mahmud A, Amore G (2008) The surprising complexity of the transcriptional regulation of the spdri gene reveals the existence of new linkages inside sea urchin's PMC and Oral Ectoderm Gene Regulatory Networks. Dev Biol.

11. Cameron R, Oliveri P, Wyllie J, Davidson E (2004) cis-Regulatory activity of randomly chosen genomic fragments from the sea urchin. Gene Expr Patterns 4: 205-213.
12. McMahon A, Flytzanis C, Hough-Evans B, Katula K, Britten R, et al. (1985) Introduction of cloned DNA into sea urchin egg cytoplasm: replication and persistence during embryogenesis. Dev Biol 108: 420-430.

13. Sodergren E, Weinstock G, Davidson E, Cameron R, Gibbs R, et al. (2006) The genome of the sea urchin Strongylocentrotus purpuratus. Science 314: 941-952.

14. Amore G, Davidson E (2006) cis-Regulatory control of cyclophilin, a member of the ETS-DRI skeletogenic gene battery in the sea urchin embryo. Dev Biol 293: 555-564.

15. Rizzo F, Fernandez-Serra M, Squarzoni P, Archimandritis A, Arnone M (2006) Identification and developmental expression of the ets gene family in the sea urchin (Strongylocentrotus purpuratus). Dev Biol 300: 35-48.

16. De Sario A, Geigl E, Bernardi G (1995) A rapid procedure for the compositional analysis of yeast artificial chromosomes. Nucleic Acids Res 23: 4013-4014. 